\section{Wie sicher ist die subkutane SIT?}

\section{Obwohl die subkutane spezifische Immuntherapie (SIT) seit langem etabliert ist, gibt es relativ wenige aussagekräftige Daten über die Häufigkeit von Nebenwirkungen in der Dosissteigerungsphase. Eine prospektive Studie sollte verlässliche Ergebnisse liefern.}

$\mathrm{n}$ vier allergologischen Zentren in Dänemark wurden insgesamt 1.038 subkutane Hyposensibilisierungen dokumentiert. Die Ärzte verwendeten unterschiedliche Steigerungsschemata über mehrere Monate. Dabei erhielten die Studienteilnehmer über 23.000 Injektionen. Verwendet wurden Allergenextrakte (ALK-Abelló) von Birke, Wiesenlieschgras, Beifuß, Hausstaubmilbe, Katze, Hund sowie Bienen- und Wespengift. 625 Patienten erhielten nur Injektionen eines Allergens an einem Tag, die übrigen zwei $(30 \%)$, drei $(21 \%)$ oder sogar vier Allergene (9\%) am selben Tag.

Es zeigten 33\% der Patienten systemische Reaktionen auf die Injektionen - bei zwei Drittel der Fälle nur ein einziges Mal. Vier von fünf der systemischen Nebenwirkungen entsprachen Schweregrad 2 mit Rhinitis und mildem Asthma. Bei 20\% der Reaktionen entwickelten die Patienten Urtikaria, Angioödem oder stärkeres Asthma (Grad 3). Bei 1\% der

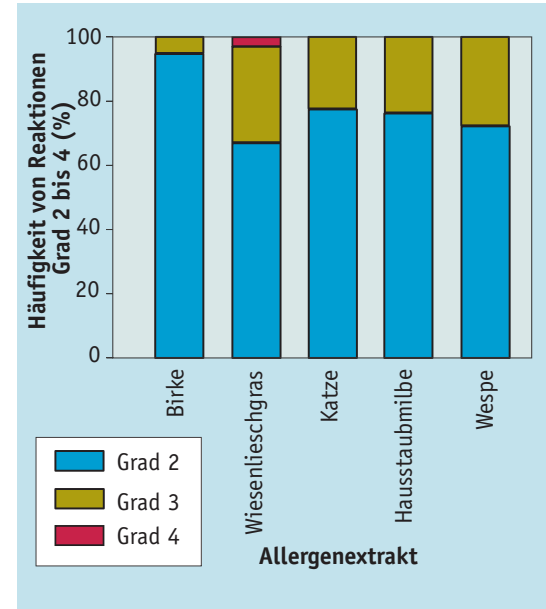

Die SIT mit Phleum-Allergenen verursacht am häufigsten schwerere Nebenwirkungen.

Fälle waren Nebenwirkungen vom Grad $4 \mathrm{zu}$ beobachten (beginnende schwere Reaktionen mit rasch zunehmendem generalisierten Juckreiz, Flush sowie Ery- them oder schweres Asthma). Sämtliche Grad-4-Reaktionen traten innerhalb der Nachbeobachtungszeit von 30 Minuten nach der Injektion auf. Nebenwirkungen vom Grad 2 oder 3 ereigneten sich in der Hälfte der Fälle erst nach einer halben Stunde.

Je nach Allergen erreichten unterschiedlich viele Patienten die Enddosis ohne Nebenwirkungen: Bei Wespengift waren es $89 \%$, gefolgt von Birke (82\%), Hausstaubmilbe (81\%) Katzenhaar (74\%) und Gräserpollen (70\%). Sämtliche Reaktionen vom Grad 4 traten bei Injektionen von Phleum pratense auf - ob allein oder kombiniert mit anderen Allergenen. Dagegen waren nach Birkenpollenapplikation fast nur Grad-2-Reaktionen zu beobachten.

Fazit: Die subkutane SIT führte in dieser Studie während der Steigerungsphase in einem von 100 Fällen zu schweren Reaktionen. Immer war dabei die Injektion von Phleum-pratense-Allergen beteiligt. Anzahl und Stärke von systemischen Nebenwirkungen fielen je nach injiziertem Allergen unterschiedlich aus.

Winther L et al. Side-effects of allergenspecific immunotherapy: a prospective multi-centre study. Clin Exp Allergy 2006; $36: 254-60$

\title{
Allergentablette etabliert sich
}

\section{Allergentabletten zur oralen Anwendung sollen bald auf den Markt kommen. Die großen klinischen Studien im Vorfeld der Einführung erlauben eine neue Bewertung der sublingualen Allergenapplikation als kausale antiallergische Behandlung.}

$\mathrm{n}$ eine doppelblinde plazebokontrollierte Studie an 51 Zentren in acht Ländern wurden insgesamt 634 Patienten mit einer Rhinokonjunktivitis aufgrund einer Gräserpollenallergie einbezogen. Sie erhielten randomisiert spätestens 16 Wochen vor Beginn der Pollensaison ohne Steigerungsphase eine lyophilisierte Tablette $\left(\right.$ Grazax $\left.^{\circledR}\right)$ mit einer Dosierung von 75.000 SQ-Einheiten Gräserpollenallergenen oder Plazebo. Der lange Vorlauf vor Beginn der Grä- serblüte sowie die Dosis von 75.000 SQ hatten sich in vorangegangenen Studien als optimale Einnahmemodalitäten erwiesen. Die Behandlung wurde über die gesamte Pollenflugzeit fortgesetzt.

Es ergaben sich nach dem ersten Behandlungsjahr ein signifikanter RückgangdesRhinokonjunktivitis-Symptomscores um 30\% ( $<<0,0001)$ und eine signifikante Reduktion des Rhinokonjunktivitis-Medikationsscores um 38\% $(\mathrm{p}<0,0001)$, jeweils im Vergleich zu
Plazebo. Auch die Zahl der Tage, an denen die Patienten keine symptomatisch wirksamen Medikamente einzunehmen brauchten und der Symptomscore unter 2 lag, erhöhte sich statistisch signifikant (53\% vs. $44 \%, \mathrm{p}<0,0001)$. Als Nebenwirkungen traten vor allem leichter Juckreiz und Schwellungen im Mundbereich auf. Schwere lokale oder systemische Reaktionen kamen nicht vor.

Fazit: Die sublinguale Immuntherapie mit einer lyophilisierten Allergentablette ist bei Patienten mit Gräserpollenallergie wirksam und sicher.

Dahl R et al. Efficacy and safety of sublingual immunotherapy with grass allergen tablets for seasonal allergic rhinoconjunctivitis. J Allergy Clin Immunol 2006; 118:

434-40 